\title{
Comparison of Structural Colors Achieved by Laser-Induced Periodic Surface Structures and Direct Laser Interference Patterning
}

\author{
Marcos Soldera ${ }^{1,2}$, Franco Fortuna ${ }^{1}$, Sascha Teutoburg-Weiss ${ }^{1}$, Stephan Milles ${ }^{1}$, Kurt Taretto ${ }^{2}$, and Andrés Fabián Lasagni ${ }^{1,3}$ \\ ${ }^{1}$ Institut für Fertigungstechnik, Technische Universität Dresden, George-Bähr-Str. 3c, 01069 \\ Dresden, Germany \\ ${ }^{2}$ PROBIEN, Dto. de Electrotecnia, CONICET, Univ. Nacional del Comahue, Buenos Aires 1400, \\ 8300, Neuquén, Argentina \\ ${ }^{3}$ Fraunhofer Institute for Material and Beam Technology IWS, Winterbergstraße 28, 01277 \\ Dresden, Germany
}

marcos.soldera@mailbox.tu-dresden.de

\begin{abstract}
Engraving surfaces with holographic features is not only used for decorative applications, but also for protecting products from counterfeit and piracy. This structural coloration can be produced on large areas at high-throughput using laser-based methods such as Direct Laser Interference Patterning and Laser-Induced Periodic Surface Structuring (LIPSS). In this contribution, both technologies were used to generate diffraction gratings on stainless steel with different processing parameters. Then, the resulting spectral reflectances were quantitatively compared and correlated to topographical characteristics determined by scanning electron microscopy and atomic force microscopy. Overall, the DLIP treated samples showed up to $42 \%$ higher diffuse reflectivity than the LIPSS samples when illuminated with a green laser diode. However, the LIPSS textures were less spectral dependent, probably due to their broader distribution of spatial periods and structure heights. These findings could also be appreciated with the naked eye as the samples patterned with LIPSS have a matte aspect with a homogeneous coloration, whereas the DLIP samples had a glossy appearance with brighter colors.
\end{abstract}

DOI: $10.2961 /$ jlmn.2020.02.2004

Keywords: structural colors, direct laser interference patterning, laser-induced periodic surface structures, optical characterization, diffraction gratings

\section{Introduction}

Usually, surfaces can be provided with a predefined coloration by applying a pigment to modify their absorbance and reflectance at given wavelengths. Even though this method is widely used, the long term stability of the color quality depends strongly on the pigment sensitivity to external physical and chemical influences, such as UV radiation, interaction with chemicals, mechanical damage or heat [1]. An alternative approach consists on producing a structural coloration through modification of the surface topography so that it interacts with the incoming light producing optical effects, such as interference, plasmonic resonances or diffraction [2-5]. In the latter case, the achieved structural color is dependent on the viewing angle, as observed in many natural cases, for example in the multiple sized structure of morpho peleides butterfly wings [6]. As the structural coloration stability depends mainly on the mechanical properties of the base material, this approach can be better suited for applications where pigments cannot withstand the environmental conditions.

Structural coloration by engraving relief gratings is used for decoration, marking and also for preventing piracy and counterfeiting [7]. Due to their upscaling potential and multifaceted application possibilities, laser based methods are commonly used to fabricate holographic features resulting from surface patterned gratings [8]. Among these techniques, Laser-Induced Periodic Surface Structuring (LIPSS) and Direct Laser Interference Patterning (DLIP) are industrial attractive methods able to engrave thousands of grooves with pitch sizes on the micro and sub-micro scale with a single laser pulse [9-14].

LIPSS consist on self-assembled nano/micro ripples formed on surfaces of a wide variety of materials, including metals, dielectrics and polymers, upon irradiation with ultra-short laser pulses in the ps and fs regime $[15,16]$. In the case of LIPSS produced on strong absorbing materials, the resulting ripples that form a quasiperiodic array with an effective spatial period close to the used laser wavelength are known as low spatial frequency LIPSS (LSFL). It is commonly accepted that these kind of LIPSS are aligned perpendicular to the radiation polarization due to the excitation of surface plasmon polaritons (SPP) [17]. LIPSS with pitch sizes smaller than half of the used laser wavelengths are called high spatial frequency LIPSS (HSFL) and they can be oriented parallel or perpendicular to the laser radiation polarization depending on the irradiated material [18]. Despite the large accumulated research on the formation of these nanostructures [19], the physical mechanism explaining 
their origin is still under debate [20-22]. Considering that gratings diffract light with wavelengths on the order of the period, the surface patterns must have spatial periods larger than a few hundreds of nanometers to yield vivid colors. Therefore, LSFLs are better-suited than HSFLs for the realization of structural colors due to their longer periods [23].

In the DLIP method, two or more laser beams are overlapped on the sample surface in order to generate an interference pattern. At the intensity maxima positions the material can be locally melted, ablated or modified, whereas at the minima positions the material remains unaffected. In this way, DLIP allows the formation of a periodic arrangement of grooves with a well-defined spatial period and with the flexibility to easily change their orientation and spatial period [12,24].

Although many published works have reported the employment of the DLIP and LIPSS techniques to produce structural colors for decoration, anti-counterfeiting or color marking [25-29], no systematic study has been reported that quantitatively and qualitatively compares the resulting coloration produced by both methods and correlates the optical properties with the topography characteristics and process parameters. This contribution aims at filling that gap by using LIPSS and DLIP methods to generate structural colors on metallic surfaces. In addition, combining optical and topography characterization methods, the optimum laser process parameters, such as fluence and pulse-to-pulse overlap, are evaluated. Moreover, the LIPSS and DLIP potential to achieve intense structural colors are quantitatively compared.

\section{Materials and methods}

\subsection{Materials}

Flat substrates of stainless steel (304) were patterned with DLIP and LIPSS structures as this material is normally used in decorative applications due to its good optical, mechanical and chemical properties. When its surface is mirror-polished has a high and almost flat reflectance above $60 \%$ in the visible spectrum [30,31]. The samples had a thickness of $0.7 \mathrm{~mm}$ with an average surface roughness $\left(R_{\mathrm{a}}\right)$ of $52 \mathrm{~nm}$. The substrates were used as received.

\subsection{DLIP structuring}

An in-house designed DLIP system equipped with a commercially available optical head (manufactured by Fraunhofer IWS, Germany) based on a ps-laser source (Edgewave PX200, Germany) emitting in the infrared $(1064 \mathrm{~nm})$ was used to structure the samples. The laser had a nominal maximum output power of $10 \mathrm{~W}$, a pulse duration of $10 \mathrm{ps}$ and the repetition rate was set to $10 \mathrm{kHz}$. The polarization direction of the laser beam is controlled by a half-wave plate. The optical DLIP head had a diffractive optical element (DOE) to split the primary beam into two sub-beams which were then parallelized by a prism. The parallel beams were afterwards overlapped onto the sample surface by a convex lens, generating the interference pattern on the sample. Adjusting the distance between DOE and prism, the angle between the interfering beams could be modified which ultimately allowed the user to adjust the spatial period of the patterned texture. Additional details of the used DLIP system have been already reported elsewhere [32]. The spatial period was set to $1.3 \mu \mathrm{m}$ for all samples, whereas the remainder of the process parameters were taken from previous experiments $[25,33,34]$. For instance, the fluence $F$ was varied between 1.5 and $2.2 \mathrm{~J} / \mathrm{cm}^{2}$, the pulseto-pulse distance $P 2 P$ was swept between 5 and $25 \mu \mathrm{m}$ and the hatch distance $H D$ was changed between 27 and $100 \mu \mathrm{m}$. The spot size was $77 \mu \mathrm{m}$ as determined from the $\mathrm{D}^{2}$ method [35]. The structured area was $5 \times 5 \mathrm{~mm}^{2}$ for all samples.

\subsection{LIPSS structuring}

The LIPSS were generated on the steel samples using linear laser radiation polarization employing the same system as for DLIP processing. The fluence was set to $3.8 \mathrm{~J} / \mathrm{cm}^{2}$, the hatch distance was varied between 60 and $120 \mu \mathrm{m}$ and the pulse-to-pulse distance was swept in the range 10 to $50 \mu \mathrm{m}$. As in the DLIP experiments, the structured area was $5 \times 5 \mathrm{~mm}^{2}$ for all samples.

\subsection{Topography characterization}

The surface topography was characterized using a non-contact optical confocal microscope (CFM) model Sensofar S Neox 3D Surface Profiler (Spain) equipped with a $150 \mathrm{X}$ objective allowing a maximum lateral and vertical resolution of $140 \mathrm{~nm}$ and $1 \mathrm{~nm}$, respectively. The samples were also characterized using a scanning electron microscope (SEM, ZEISS Supra 40VP, Germany) at an operating voltage of $8.0 \mathrm{kV}$.

\subsection{Optical characterization}

To measure the intensity of the diffuse, i.e. nonspecular, reflectivity, a self-developed characterization system was used (Fig. 1). The collimated beam emitted by a low power laser diode was reduced to a diameter of approximately $2 \mathrm{~mm}$ by a set of lenses, so that it could fit through the $2.5 \mathrm{~mm}$ hole of a laser-drilled monocrystalline solar cell (SunPower, USA). Then, the beam was directed to the sample, which was placed at $2 \mathrm{~cm}$ from the solar cell and was tilted $5^{\circ}$. While the diffracted pattern was reflected back to the front surface of the solar cell, the specularly reflected beam was blocked (not shown in Fig. 1). In this way, only the diffusely reflected light was incident on the solar cell. The area of the solar cell was $41 \times 125 \mathrm{~mm}^{2}$, which is large enough to collect the main diffracted patterns by the laser-treated samples. Although there might be some randomly scattered light with very wide angles $\left(>60^{\circ}\right)$ that may not hit the solar cell, it is considered that the amount of such radiation is negligible. Furthermore, shadowing losses were prevented by the interdigitated back contact (IBC) of the solar cell, whereas the reflectance of the solar cell was minimized due to its pyramidal texture combined with an anti-reflective coating. Finally, the photocurrent delivered by the solar cell was measured with a transconductance amplifier and a voltmeter. As the solar cell is kept under short circuit conditions by the external circuitry, the operational amplifier operated in the linear region and the laser intensity was constant throughout the experiments, the measured voltage signal is directly proportional to the 
intensity of the reflected light. The measured signal for each sample was normalized to the intensity corresponding to the specularly reflected light from a flat steel plate. Three different laser diodes with wavelengths of $450 \mathrm{~nm}$, $532 \mathrm{~nm}$ and $635 \mathrm{~nm}$ were used to obtain spectral information of the samples.

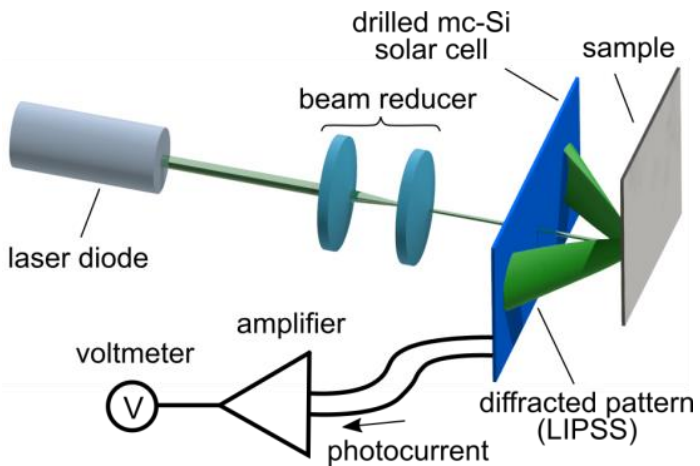

Fig. 1 Schematic representation of the setup used to measure the diffuse reflectivity. The zero order reflected beam (specular reflection) is blocked (not shown in the figure for simplicity).

The spatial distribution of the reflected patterns was recorded with an in-house developed imaging system coupled to a CCD camera. Further details can be found elsewhere [34].

\section{Results and discussion}

\subsection{Surface topography}

The SEM images of Fig. 2 show the surface topography of three DLIP-treated samples with the same hatch distance and pulse-to-pulse feed, namely $82 \mu \mathrm{m}$ and $20 \mu \mathrm{m}$, respectively. In turn, the laser fluence was set to $1.5 \mathrm{~J} / \mathrm{cm}^{2}, 1.8 \mathrm{~J} / \mathrm{cm}^{2}$ and $2.2 \mathrm{~J} / \mathrm{cm}^{2}$, for the samples shown in Fig. 2a), b) and c), respectively.

The SEM images show that the line-like textures have an overall good structure uniformity in the three samples and that the grooves have a periodic distribution with a spatial period of $1.3 \mu \mathrm{m}$, as expected. Aside from the DLIP grooves, LIPSS are also visible which can be identified as HSFL, as they are aligned parallel to the radiation polarization (see arrow in Fig. 2a) and their spatial period is approximately $300-500 \mathrm{~nm}$, i.e. less than half of the laser wavelength. In contrast, due to the relatively low chosen fluence, LSFL are not observed on any sample. As the fluence increases, the depth of the grooves becomes larger and the HSFL are better defined, although more molten material becomes visible.

The surface morphology of a LIPSS-treated sample can be seen in the SEM micrograph of Fig. 3. This sample was structured with a hatch distance of $80 \mu \mathrm{m}$, a pulseto-pulse feed of $30 \mu \mathrm{m}$ and a fluence of $3.8 \mathrm{~J} / \mathrm{cm}^{2}$. Here, both type of LIPSS are visible and they are uniformly distributed over the surface. While the LSFL have a period between 800 and $1000 \mathrm{~nm}$, the HSFL have a period in the range $300-500 \mathrm{~nm}$, as observed in the DLIP structured samples. Not surprisingly, the presence of both LSFL and HFSL with their relative broad spatial periods distribution in comparison to the DLIP textures, yields also a less uniform structure height over the whole surface.

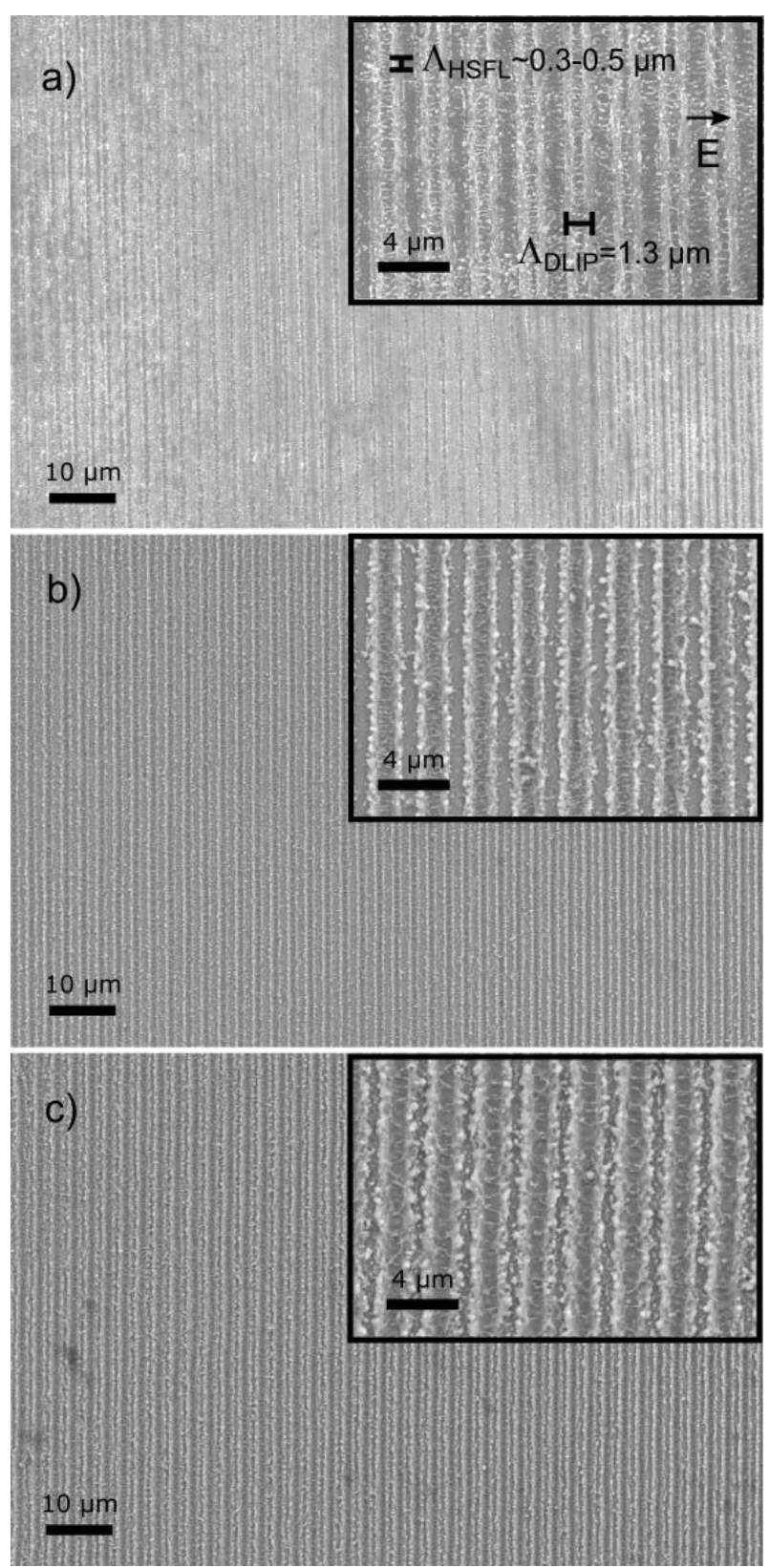

Fig. 2. SEM images of DLIP structured samples with a hatch distance of $82 \mu \mathrm{m}$ and pulse-to-pulse feed of $20 \mu \mathrm{m}$, with varying fluence: a) $1.5 \mathrm{~J} / \mathrm{cm}^{2}$, b) $1.8 \mathrm{~J} / \mathrm{cm}^{2}$ and c) $2.2 \mathrm{~J} / \mathrm{cm}^{2}$. The arrow in the insert in a) points at the laser radiation polarization.

Fig. 4 shows exemplarily the surface topography of a) DLIP $\left(H D=100 \mu \mathrm{m}, P 2 P=20 \mu \mathrm{m}, F=1.8 \mathrm{~J} / \mathrm{cm}^{2}\right)$ and b) LIPSS $\left(H D=100 \mu \mathrm{m}, P 2 P=30 \mu \mathrm{m}, F=3.8 \mathrm{~J} / \mathrm{cm}^{2}\right)$ treated samples measured with CFM and their corresponding FFT (fast Fourier transform) intensity spectra b) and d), respectively. It can be seen that in the FFT spectrum of the DLIP sample, the intensity peaks are clearly defined and equidistantly spaced, resembling the spectrum of perfectly ordered diffraction gratings. The extracted period from the FFT spectrum is $1.29 \pm 0.02 \mu \mathrm{m}$ for all the DLIP samples. Nevertheless, a background noise can be detected for small frequencies which can be correlated to texture defects. Despite the FFT spectrum of LIPPS texture has its characteristic double-crescent shape as observed in many previous reports [36-38], a relatively large background noise can be observed for those frequencies contained within the dou- 
ble-crescent. Correlating the spatial frequencies of all the LIPSS-treated samples with the corresponding spatial periods, the Fourier analysis revealed that the distribution of periods of the LIPSS did not change significantly with the process parameters. Namely, the LIPSS textures have an average dominant period of $930 \pm 30 \mathrm{~nm}$ and an approximate period range of $800-1100 \mathrm{~nm}$, in agreement with the periods observed in the SEM micrographs.
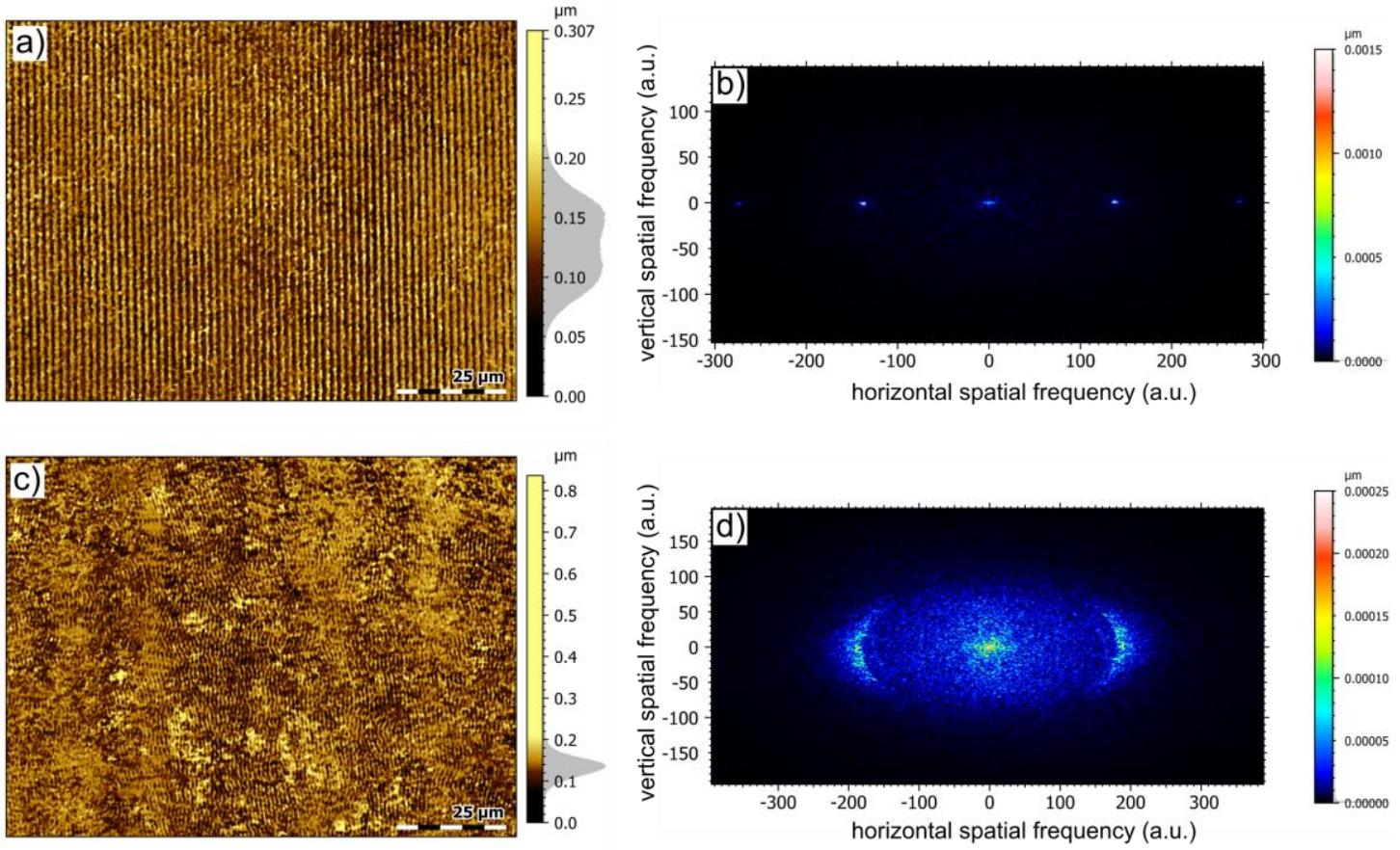

Fig. 4 CFM topography images of a) DLIP and c) LIPSS treated samples and their FFT spectra b) and d), respectively.

\subsection{Optical properties}

Upon illuminated with a point-like white light source, all the laser-structured samples present a structural "rainbow" coloration, that strongly depends on the viewing angles as occurs in conventional diffracting gratings. Fig. 5 shows two photographs of the DLIP and LIPSS textured steel plates. It can be seen that the LIPSS samples exhibit a matte finish with homogenous coloration, whereas the DLIP treated areas have a glossy appearance and brighter colors. As these observations are inherently subjective, the reflected light by the samples was characterized spatially and spectrally to obtain more significant data that allows an objective comparison between them.

The diffraction patterns shown in Fig. 6 were recorded with an imaging system based on a low power laser diode with a wavelength of $532 \mathrm{~nm}$ and a CCD camera [34]. Fig. 6a) corresponds to a selected DLIP sample $\left(H D=100 \mu \mathrm{m}, P 2 P=20 \mu \mathrm{m}, F=1.8 \mathrm{~J} / \mathrm{cm}^{2}\right)$, where the

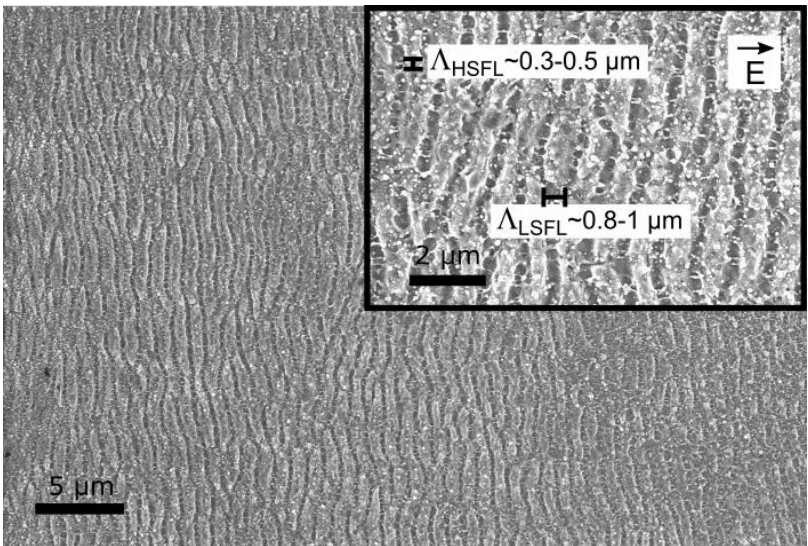

Fig. 3 SEM image of a steel sample structured with LIPSS at a hatch distance of $80 \mu \mathrm{m}$, a pulse-to-pulse feed of $30 \mu \mathrm{m}$ and a fluence of $3.8 \mathrm{~J} / \mathrm{cm}^{2}$. Both LSFL and HSFL can be identified.

The arrow in a) points at the laser radiation polarization.

diffraction peaks up to the second order are detected with the used imaging optics. In turn, the pattern in Fig. 6b) belongs to a LIPSS treated area $(H D=100 \mu \mathrm{m}$, $P 2 P=30 \mu \mathrm{m}, F=3.8 \mathrm{~J} / \mathrm{cm}^{2}$ ) showing its distinctive double-crescent shape. As expected, the recorded patterns can be directly correlated to the FFT spectra shown in Fig 4 , but in the CCD images a higher signal-to-noise ratio can be measured allowing a better comparison between the samples. The intrinsic narrow angular distribution of the intensity within the diffraction peaks of the DLIP samples might explain their glossy aspect. In contrast, the LIPSS textures diffract light with a more widespread angular distribution (as observed in Fig. 6b) explaining the matte appearance.

Both images shown in Fig. 6 were captured with the same integration time, which was set at its maximum where the double-crescent signal starts to saturate. Contrarily, the diffraction peaks of the DLIP samples are already saturated hinting at a much stronger diffracted in- 
tensity in contrast to the LIPSS structured areas. Therefore, a self-developed optical system was used to measure the intensity of the diffused reflected light from the samples that permits a quantification of the structural coloration.

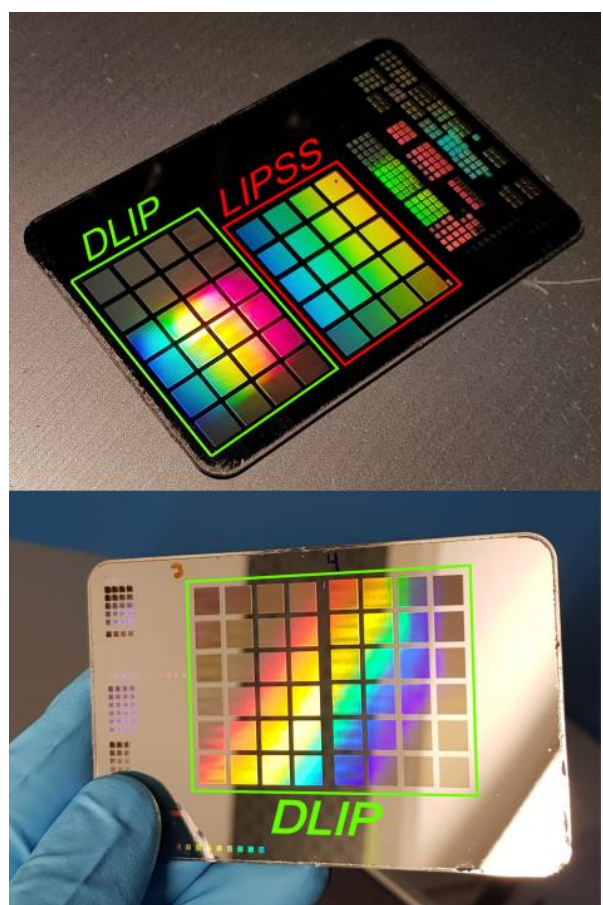

Fig. 5 Photographs of LIPSS and DLIP treated samples (as labeled in the figure) showing the structural coloration. and pulse-to-pulse feed is shown in a matrix form in Fig. 7. The rows of the matrix indicate different fluence values during the laser processing and the columns different measured wavelengths, i.e. reflected colors, as labeled in the figure. The highest intensities were recorded for those samples structured at higher fluences and higher $P 2 P$ values, reaching an absolute maximum of 0.4 for the green color at $F=2.2 \mathrm{~J} / \mathrm{cm}^{2}, \quad P 2 P=25 \mu \mathrm{m}$ and $H D=73 \mu \mathrm{m}$. It is assumed that the highest color intensities are achieved at the highest fluence and $P 2 P$ due to the better texture homogeneity and uniform distribution of the structure height around $100 \mathrm{~nm}$, as revealed from SEM and CFM images. The results also show that the green color has the highest intensities, whereas the reflected blue color is less intense in practically all samples
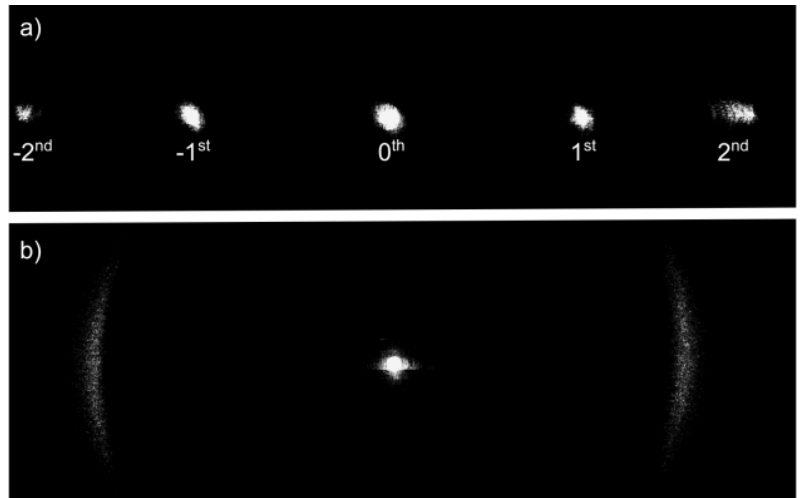

Fig. 6 Diffraction patterns recorded with a CCD camera of typical a) DLIP and b) LIPSS treated samples.

The normalized diffuse reflected intensity of all the DLIP structured samples as function of hatch distance

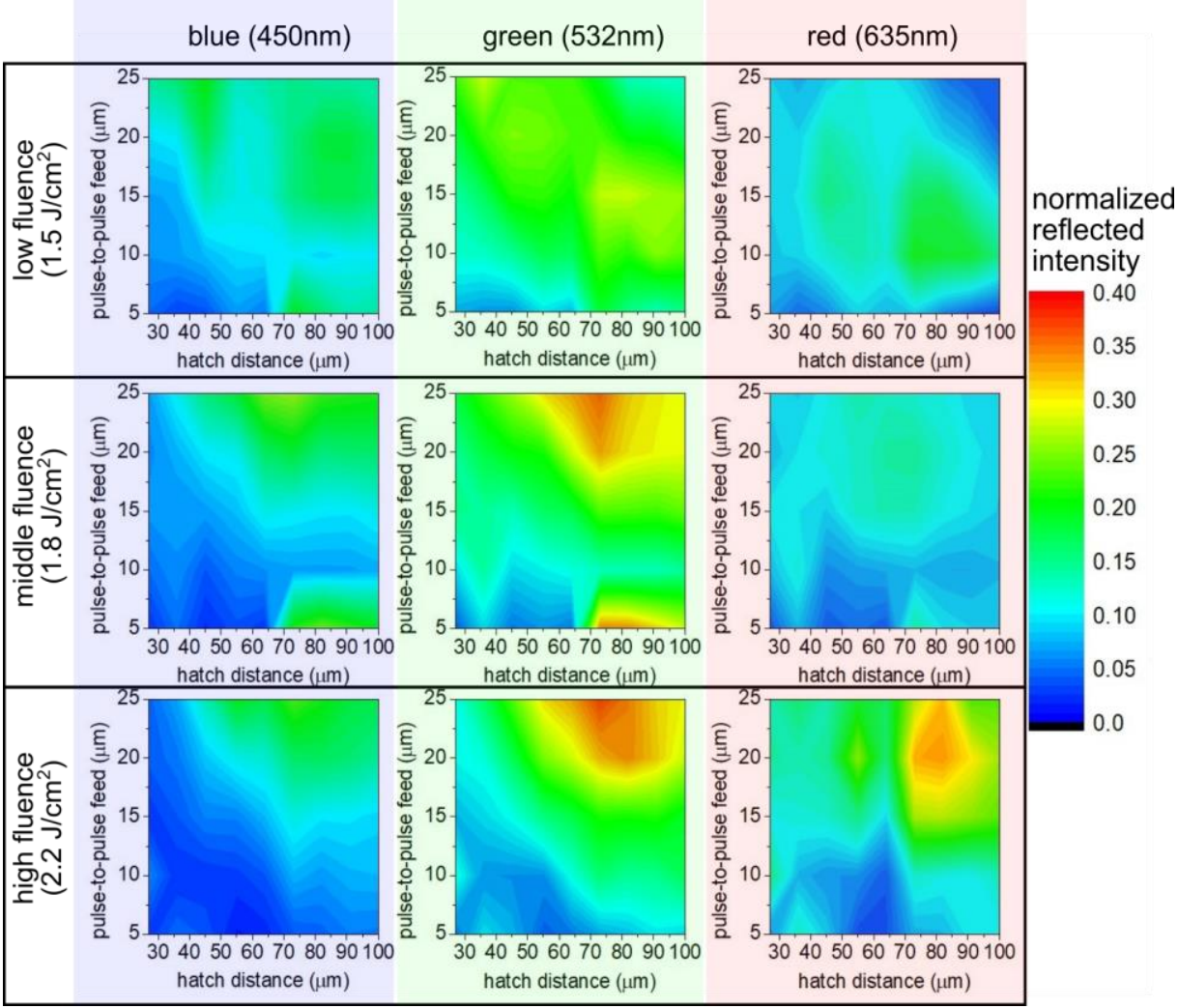

Fig. 7 Normalized diffuse reflectance of the DLIP structured samples as function of processing conditions, i.e. hatch distance, pulse-topulse feed and fluence, as well as the wavelength of the illumination source. 

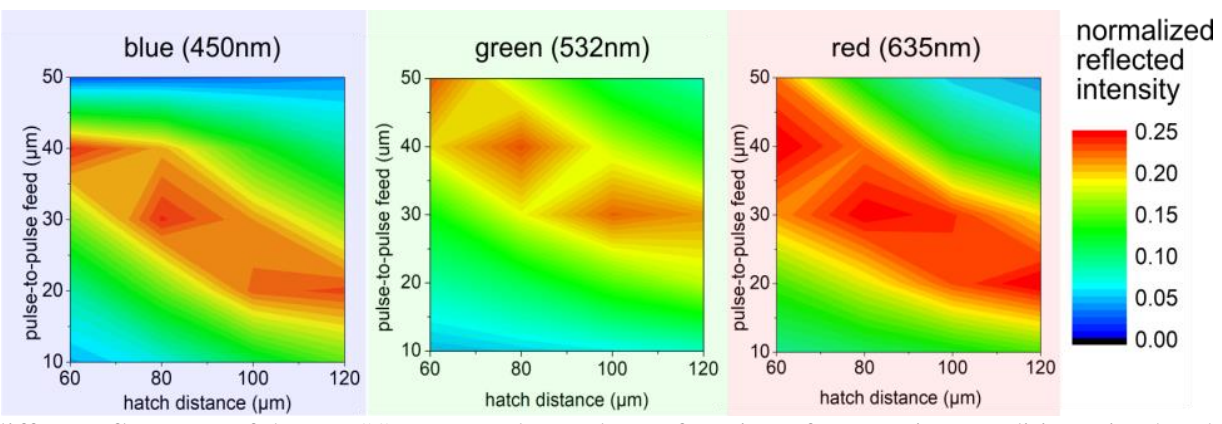

Fig. 8 Normalized diffuse reflectance of the LIPSS structured samples as function of processing conditions, i.e. hatch distance and pulseto-pulse feed, as well as the wavelength of the illumination source.

Fig. 8 shows the normalized diffuse reflected intensity of all LIPSS treated samples, when illuminated with the three different laser diodes, i.e. colors. In this case, the three sub-plots show a region with a diagonal band shape, where the maximum intensities are found. The absolute maximum of 0.25 is reached for the red color in a relatively broad region allowing for a more flexible structuring parameters selection than for the DLIP process. This is due to the fact, that as the DLIP method produces periodic grooves with a well-defined spatial period, the surface topography must be very homogeneous to achieve the desired diffraction grating effect. Thus, the quality of the structural coloration in DLIP textures is more sensitive to the process parameters. In general, it was observed in the SEM and CFM images that applying high accumulated fluences, i.e. small pulse-to-pulse feed and hatch combined with high fluences, leads to a large amount of recast material that tends to destroy the periodicity of the texture. This degradation of the periodicity can be noticed by the random distribution of structure heights as well as a non-uniform shape of the DLIP grooves. Likewise, applying low accumulated fluences, for instance by irradiating with low fluences and setting high pulse-topulse feeds and hatch distances, induces also a poor texture homogeneity due to the gaussian distribution of the radiation intensity. Therefore, the regions in Fig. 7 where the diffuse reflectance of the DLIP samples was maximized correspond to those samples with the highest texture homogeneity. In contrast, the measured reflectance on the LIPSS treated surfaces also shows a less spectral dependence than in the DLIP samples, which can be attributed to a broader distribution of the LIPSS structure heights and spatial periods (or equivalently, a broad-band distribution of spatial frequencies as revealed by the FFT spectrum of LIPSS topography, Fig. 4). However, these intensities are, in the best case, $12 \%, 42 \%$ and $24 \%$ lower, compared to the DLIP structures, for blue, green and red colors, respectively.

\section{Conclusions}

In this contribution, an approach to systematically compare colored surfaces achieved by engraving relief gratings using DLIP and LIPSS technologies was presented. The optical appearance of the stainless steel samples processed with both technologies exhibited the typical rainbow coloration characteristic of diffraction gratings. The samples could be clearly differentiated with the naked eye, as the LIPSS samples had a matte appearance, while the DLIP structured areas had a glossy aspect. This difference might be attributed to their inherent diffraction patterns. On the one hand, the DLIP textures had a welldefined spatial period yielding a discrete set of highly intense diffraction peaks, upon illuminating them with a coherent light beam. On the other hand, the LIPPS features formed a quasi-periodic texture, whose diffraction pattern resembles a double-crescent shape with a relatively broad spatially distributed intensity.

The optical characterization revealed that the processing parameters, namely hatch distance, pulse-to-pulse feed and laser fluence, have a strong impact in the measured diffuse reflectance. In contrast, the laser parameters did not induce any significant change in the spatial periods of DLIP or LIPSS samples. A relation between reflectivity and quality of the topography could be established, hinting at higher reflected intensities for those samples with a better texture homogeneity and uniform structure depths around $100 \mathrm{~nm}$. Likewise, the FFT spectra corresponding to the topographical data measured with CFM could be directly correlated to the diffraction patterns of DLIP and LIPSS samples recorded with a CCD camera. Comparing the diffuse reflectance results, a significant $60 \%$ higher maximum intensity was recorded for the DLIP structures compared with the LIPSS grooves. However, the LIPSS textures showed a nearly constant spectral response, which could be an advantage for some applications.

\section{Acknowledgments}

This work was carried out in the framework of the Reinhart Koselleck project (323477257), which has received funding from the German Research Foundation (German: Deutsche Forschungsgemeinschaft DFG). M.S acknowledges the support of the Alexander von Humboldt Foundation.

\section{References}

[1] E. Ghelardi, I. Degano, M. P. Colombini, J. Mazurek, M. Schilling, H. Khanjian, and T. Learner: Dyes Pigments, 123, (2015) 396.

[2] J. Orava, N. Heikkila, T. Jaaskelainen, and J. Parkkinen: J. Opt. Soc. Am. A, 25, (2008) 2901.

[3] T. Xu, H. Shi, Y.-K. Wu, A. F. Kaplan, J. G. Ok, and L. J. Guo: Small, 7, (2011) 3128.

[4] S. Kinoshita, S. Yoshioka, and J. Miyazaki: Rep. Prog. Phys., 71, (2008) 076401. 
[5] R. Zhou, T. Huang, Y. Lu, and M. Hong: Appl. Sci., 8, (2018) 1716.

[6] P. Vukusic, J. R. Sambles, C. R. Lawrence, and R. J. Wootton: P. Roy. Soc. B-Biol. Sci., 266, (1999) 1403.

[7] R. Y. Shah, P. N. Prajapati, and Y. K. Agrawal: J. Adv. Pharm. Technol. Res., 1, (2010) 368.

[8] H. Liu, W. Lin, and M. Hong: APL Photonics, 4, (2019) 051101.

[9] J.-M. Guay, A. C. Lesina, J. Baxter, G. Killaire, L. Ramunno, P. Berini, and A. Weck: Adv. Opt. Mater., 6, (2018) 1800189.

[10] B. Dusser, Z. Sagan, H. Soder, N. Faure, J.-P. Colombier, M. Jourlin, and E. Audouard: Opt. Express, 18, (2010) 2913.

[11] G. Li, J. Li, Y. Hu, C. Zhang, X. Li, J. Chu, and W. Huang: Appl. Phys. A, 118, (2015) 1189.

[12] A. F. Lasagni: Adv. Opt. Tech., 6, (2017) 265.

[13] R. J. Peláez, E. Rebollar, R. Serna, C. AcostaZepeda, P. Saavedra, J. Bonse, and E. HaroPoniatowski: J. Phys. D: Appl. Phys., 52, (2019) 225302.

[14] I.-Y. Park, S. Ahn, Y. Kim, H.-S. Bae, H.-S. Kang, J. Yoo, and J. Noh: Opt. Express, 25, (2017) 14644.

[15] S. Höhm, M. Herzlieb, A. Rosenfeld, J. Krüger, and J. Bonse: Appl. Surf. Sci., 374, (2016) 331.

[16] R.-A. Barb, C. Hrelescu, L. Dong, J. Heitz, J. Siegel, P. Slepicka, V. Vosmanska, V. Svorcik, B. Magnus, R. Marksteiner, M. Schernthaner, and K. Groschner: Appl. Phys. A, 117, (2014) 295.

[17] J. E. Sipe, J. F. Young, J. S. Preston, and H. M. van Driel: Phys. Rev. B, 27, (1983) 1141.

[18] J. Bonse, S. Höhm, S. V. Kirner, A. Rosenfeld, and J. Krüger: IEEE J. Sel. Top. Quant., 23, (2017) 9000615.

[19] J. Bonse, J. Krüger, S. Höhm, and A. Rosenfeld: J. Laser. Appl., 24, (2012) 042006.

[20] X.-F. Li, C.-Y. Zhang, H. Li, Q.-F. Dai, S. Lan, and S.-L. Tie: Opt. Express, 22, (2014) 28086.

[21] X. Sedao, M. V. Shugaev, C. Wu, T. Douillard, C. Esnouf, C. Maurice, S. Reynaud, F. Pigeon, F.
Garrelie, and L. V. Zhigilei: ACS Nano, 10, (2016) 6995.

[22] S. N. Volkov, A. E. Kaplan, and K. Miyazaki: Appl. Phys. Lett., 94, (2009) 041104.

[23] J. Yao, C. Zhang, H. Liu, Q. Dai, L. Wu, S. Lan, A. V. Gopal, V. A. Trofimov, and T. M. Lysak: Appl. Surf. Sci., 258, (2012) 7625.

[24] F. Rößler, T. Kunze, and A. F. Lasagni: Opt. Express, 25, (2017) 22959.

[25] B. Voisiat, W. Wang, M. Holzhey, and A. F. Lasagni: Sci. Rep., 9, (2019) 1.

[26] S. Storm, S. Alamri, M. Soldera, T. Kunze, and A. F. Lasagni: Macromol. Chem. Phys., 220, (2019) 1900205.

[27] N. Livakas, E. Skoulas, and E. Stratakis: OptoElectron. Adv., 3, (2020) 190035.

[28] J. Long, P. Fan, M. Zhong, H. Zhang, Y. Xie, and C. Lin: Appl. Surf. Sci., 311, (2014) 461.

[29] H. Wu, Y. Jiao, C. Zhang, C. Chen, L. Yang, J. Li, J. Ni, Y. Zhang, C. Li, Y. Zhang, S. Jiang, S. Zhu, Y. Hu, D. Wu, and J. Chu: Nanoscale, 11, (2019) 4803.

[30] R. A. Castelli, P. D. Persans, W. Strohmayer, and V. Parkinson: Corros. Sci., 49, (2007) 4396.

[31] B. Karlsson and C. G. Ribbing: J. Appl. Phys., 53, (1982) 6340.

[32] M. Soldera, Q. Wang, F. Soldera, V. Lang, A. Abate, and A. F. Lasagni: Adv. Eng. Mater., 22, (2020) 1901217.

[33] A. I. Aguilar-Morales, S. Alamri, and A. F. Lasagni: J. Mater. Proc. Technol., 252, (2018) 313.

[34] S. Teutoburg-Weiss, B. Voisiat, M. Soldera, and A. F. Lasagni: Materials, 13, (2020) 53.

[35] Y. C. Kiang and R. W. Lang: Appl. Opt., 22, (1983) 1296.

[36] P. Gregorčič, M. Sedlaček, B. Podgornik, and J. Reif: Appl. Surf. Sci., 387, (2016) 698.

[37] S. Gräf and F. A. Müller: Appl. Surf. Sci., 331, (2015) 150.

[38] M. Ardron, N. Weston, and D. Hand: Appl. Surf. Sci., 313, (2014) 123.

(Received: June 4, 2020, Accepted: July 28, 2020) 